\title{
El desarrollo del neoaprendizaje de los jóvenes estudiantes Nousmedi@ en su trayecto educativo hacia la formación profesional
}

\author{
The Development of Neolearning of the Nousmedi@ Student Youth in Their \\ Educational Path Towards Professional Training
}

\section{O desenvolvimento do neo-aprendizado de jovens estudantes Nousmedi @ em sua jornada educacional em direção à formação profissional}

Cirila Banda Luna

Universidad Pedagógica Nacional, Unidad 021, México

balucir@hotmail.com

https://orcid.org/0000-0002-9269-5043

\section{Resumen}

Tomando en cuenta el concepto de neoaprendizaje, el cual explica cómo aprenden las nuevas generaciones cuando interactúan en los medios digitales, y abre el camino para reconceptualizar el aprendizaje de los estudiantes con hábitos de estudio de carácter cibernético orientado a las búsquedas virtuales, se emprendió un proceso exploratorio de 344 alumnos de sexto de primaria en el año 2007. De este grupo de estudiantes observados en la fecha ya indicada, 97 se identificaron como Nousmedi@: sujetos con rasgos característicos del aprendizaje de inicios del siglo XXI. Después de 10 años, se buscó en diversas universidades y departamentos de educación a los 97 alumnos, ahora jóvenes profesionales, hasta lograr localizar a algunos de ellos en el grado o carrera que estuvieran desempeñando. Esto para conocer sus opiniones y experiencias en su trayecto educativo hacia el nivel profesional. Si bien se identificaron a 66 de los 97, solo se pudo entrevistar a nueve de ellos. Por un lado, sus respuestas manifiestan cómo la situación educativa continúa hoy en día tan 


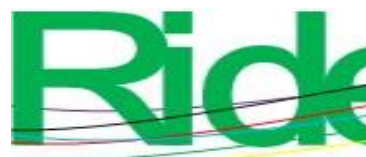

Revista Iberoamericana para la

Investigación y el Desarrollo Educativo

ISSN $2007-7467$

cuestionable en el manejo y uso de los medios digitales con prácticas de enseñanza que aletargan el aprendizaje. Y por el otro, se puede observar cómo la utilización cada vez más avanzada de los recursos cibernéticos en un sentido autoformativo posibilita una mejor situación profesional o laboral.

Palabras clave: enseñanza, formación de docentes, neoaprendizaje, Nousmedi@.

\section{Abstract}

Taking into account the concept of neolearning, which explains how new generations learn when they interact in digital media, and which opens the way to reconceptualize the learning of students with cybernetic study habits oriented to virtual searches, an exploratory process of 344 students in sixth grade was carried out in 2007. Of this group of students observed on the date already indicated, 97 identified themselves as Nousmedi@: subjects with characteristic features of early 21 st century learning. After 10 years, 97 students, now young professionals, were searched in various universities and education departments until some of them were found in the degree or career they were performing. This to know their opinions and experiences in their educational journey towards the professional level. Although 66 of the 97 were identified, only nine of them could be interviewed. On the one hand, their responses show how the educational situation continues today so questionable in the management and use of digital media with teaching practices that let you learn. And on the other, it can be seen how the increasingly advanced use of cyber resources in an autoformative sense enables a better professional or work situation.

Keywords: teaching, teacher education, neolearning, Nousmedi@.

\section{Resumo}

Levando em conta o conceito de neo-aprendizagem, que explica como as novas gerações aprendem quando interagem nas mídias digitais, e abre caminho para reconceitualizar a aprendizagem dos alunos com hábitos de estudo cibernético orientados para pesquisas virtuais, realizamos um processo exploratório de 344 alunos da sexta série em 2007. Desse grupo de alunos observado na data já indicada, 97 se identificaram como Nousmedi @: sujeitos com características características da aprendizagem do início do século XXI. Após 10 anos, 97 estudantes, agora jovens profissionais, foram revistados em várias universidades 
e departamentos de educação até conseguirem localizar alguns deles no grau ou na carreira que estavam realizando. Isso para saber suas opiniões e experiências em sua jornada educacional em direção ao nível profissional. Embora 66 dos 97 tenham sido identificados, apenas nove deles puderam ser entrevistados. Por um lado, suas respostas mostram como a situação educacional continua hoje tão questionável no gerenciamento e uso da mídia digital com práticas de ensino que permitem aprender. Por outro lado, pode-se ver como o uso cada vez mais avançado dos recursos cibernéticos em um sentido autoformativo permite uma melhor situação profissional ou de trabalho.

Palavras-chave: ensino, formação de professores, neo-aprendizagem, Nousmedi@. Fecha Recepción: Diciembre 2018

Fecha Aceptación: Septiembre 2019

\section{Introducción}

El caso inaplazable de la educación, por su estado de crisis permanente ante los avances tecnológicos, y a razón de que la información y la comunicación se encuentran al alcance de las nuevas generaciones, ha desafiado a las instituciones educativas, como la escuela, la familia, los hospitales y las religiones, y ha dejado inestable el control educativo. La evidencia de esto está en las problemáticas que se suscitan en lo formal, y en la formación para la vida, entre padres e hijos, maestros y estudiantes, médicos y pacientes, iglesias y feligreses. Se observa en las relaciones que se quebrantan, cuando el encuentro entre ambos es desde lo "obligatorio" o el "deber ser" con argumentos autoritarios, que este autoritarismo o liberación extrema aleja a la persona de sí misma, ya sea por incomodidad, desvalorización o estrategia de escape, lo cual es más evidente en la relación escuela-familia, tal y como lo menciona Quinta (1993). Entonces, sin reflexionar, se dejan al margen de influencias externas en el mundo digital.

En este análisis, los estudiantes son el centro de la preocupación educativa; reflejan en sus formas de estudio una formación insuficiente en valores, el olvido del cuidado de un cuerpo saludable o en la dispersión de sus conductas por la indiferencia, aislamiento y desintegración de grupos afines; en fin, la forma en cómo están siendo poco moldeables educativamente. Los jóvenes encuentran a su paso el tener que "comprender lo que está cambiando y lo que en buena parte ya ha cambiado, que no es otra cosa que el territorio 


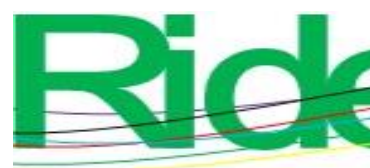

\section{Revista Iberoamericana para la Investigación y el Desarrollo Educativo ISSN $2007-7467$}

mismo" donde ellos se desenvuelven cotidianamente (Benasayag, 2015, p. 31). En la mayoría de los casos, lo que aprehenden virtualmente por medio de las computadoras, celulares, tabletas electrónicas, entre otros aparatos, no se enlaza con la vida tangible, como los adultos esperan. De ahí que entre ellos forman relaciones interactivas y fugaces sin consolidar grupos determinados, por lo que no se aprecian en comunidad para identificarse en sus nuevos rasgos y complejizan su orientación socioeducativa ante la mirada docente.

En esta complejidad que consume a la escuela hoy en día, la disolución del esquema de enseñanza como transmisión de conocimientos en el aula frente al mundo interactivo que la tecnología ha generado, debido a que tiene más información el estudiante por navegar en Internet más número de horas que el profesor, el aprendizaje se ha limitado a libros de texto básicos o materiales conocidos para la enseñanza, y por tanto, queda inconclusa la construcción de significados. Para ello, es necesario resolver este problema pedagógico y hacer una pauta para reubicar el acto de estudiar, la estrategia de articular, resolver esta tensión entre lo que se encuentran en la Red y lo que deben aprender y se encuentra indicado en la currícula escolar.

La estrategia de enseñanza que aquí resulta central es reconocer las características de esta nueva generación Nousmedi@, comprendiendo la capacidad de sus integrantes de crear nuevas lógicas de análisis de los contenidos escolares y su formación para la vida, construyéndolas con ellos, no alienando su percepción de la realidad. Tarea que se convierte en una premisa para los educadores: saber qué hacer con la diversidad de información que presentan los jóvenes en clase e identificar su aportación a nuevas explicaciones de contenidos. Principalmente, considerar a los estudiantes y toda aquella información que aprehenden extraclase y su potencial académico. También aprender otras formas de atraer el interés y atención al contenido de análisis, sin orillar al alumno a alejarse y desmotivarlo con disciplinamiento y más bien, abordar e integrar sus acciones en los dispositivos digitales. Al respecto, Benasayag (2015) subraya lo siguiente:

Cuando uno siente que un videojuego o una comunicación (en general, compulsiva e inútil) requieren de su atención, estamos hablando de una atención que es simétricamente contraria a la que necesitamos, por ejemplo, para los estudios o toda otra forma de pensamiento complejo. Estos mecanismos funcionan sobre una serie de bucles rápidos, que poco a poco dificultan la posibilidad de prácticas que requieren otro tipo de temporalidad 


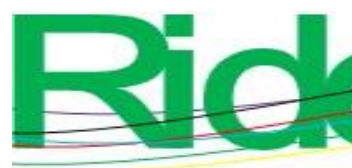

Revista Iberoamericana para la Investigación y el Desarrollo Educativo ISSN $2007-7467$

y atención. Los circuitos de la distracción se articulan (demasiado) bien con los circuitos dopamínicos de recompensa, creando de esta dependencia (que no es exactamente lo mismo que una adicción) y estados de falta química, como en cualquier otra dependencia molecular (p. 96).

$\mathrm{Al}$ reconocer que se tiene en frente a un sujeto con una distancia entre su pensar y su mundo, entre él mismo y lo que acontece en las aulas, es preciso identificar eso que le atrapa el pensamiento y la inquietud o pasividad, llevarlo de una información a otra para darle vida a través de contenidos escolares que despierten la reflexión de pensarse en el mundo auténtico. De acuerdo con Freire (1997):

Respetar la lectura de mundo del educando significa tomarla como punto de partida para la comprensión del papel de curiosidad, de modo general, y de la humana, de modo especial, como uno de los impulsos formadores de la producción de conocimiento (p. 117).

Es colocarse como responsable del rol de educar y formar a su alumno, hijo, paciente o feligrés, en la atención a lo que percibe directamente y lo que le dará mayor sentido a lo real sobre lo virtual.

Las generaciones jóvenes, quienes van apropiándose de afinidades que surgen de las redes que se articulan a través de la búsqueda, donde los intereses pueden ser los mismos, pero la navegación virtual tener diferentes direcciones, se identifican con el hecho de que "la personalización es una estrategia básica de los cinco principales gigantes de Internet Yahoo, Google, Facebook, Youtube y Microsoft Live-" (Pariser, 2017, p. 17), conocidos como buscadores en redes digitales con marcas registradas. En la burbuja de los filtros personalizados de cada internauta se provoca la atención para clickear en los anuncios relacionados con los artículos que se buscaron anteriormente y que se combinan según los temas también antes explorados en la interfaz, situación que escapa de la comprensión cultural de los adultos, por lo que se vuelve imprescindible intervenir en este mundo digital o transformar nuestro paradigma educativo como educadores.

El antecedente del presente trabajo es la búsqueda de la conceptualización de la ya referida generación Nousmedi@. Dicho antecedente se inició como una investigación exploratoria en los años 2006-2008, realizada por Banda (2013), uno de los primeros esfuerzos de acercamiento a esta problemática. Ahora bien, respecto a estos sujetos observados en ese entonces, población por conveniencia, quienes eran niños de sexto grado 


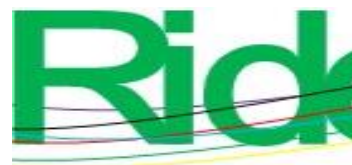

Revista Iberoamericana para la Investigación y el Desarrollo Educativo ISSN $2007-7467$

y actualmente son jóvenes, hoy en día surgen los siguientes cuestionamientos: ¿Cuál ha sido el avance de los 97 alumnos identificados en 2008 en su trayecto escolar? ¿Se encuentran en grado universitario, egresados con formación profesional o no lograron continuar sus estudios? Sobre todo, porque han sido influidos desde lo que para Aronowitz, Martinsons y Menser (1998) se conoce como la tecnociencia y la cibercultura, sin duda poco reflexionadas en las aulas. Ahora este mundo forma parte de una realidad en la que se desenvuelven, teniendo como referencias accesibles lo que encuentran en internet al margen del mundo adulto, quien se encuentra ocupado.

Partimos de la premisa de que la generación Nousmedi@ aprende a partir de su interacción virtual, lo que llamamos neoaprendizaje. Este se reconoce como el encuentro entre los diversos y consensados contenidos que comparten previamente en las redes virtuales y llevan a las actividades escolares en forma verbal, impresos o en objetos de aprendizaje que ellos mismos acuerdan y, con ello, facilitan la enseñanza en las aulas, si así lo permite el docente. La escuela, como espacio educativo donde aparece el estudiante con cada vez mayor cantidad de herramientas digitales, necesita acceder a la integración del uso de dispositivos que impulsan el desarrollo cognitivo, socioemocional y tecnocultural. En ese sentido las aportaciones de Benasayag (2017) resultan valiosas, pues explica cómo, al llegar al aula, el estudiante necesita sentir en su cuerpo el "cerebro aumentado", que se desarrolla y aprende a tomar decisiones posibles sobre la potencia de lo nuevo extraída del mundo virtual, donde pareciera que todo es accesible, a no ser que se topen contra una realidad estructurada en forma diferente a lo que ellos imaginan.

Frente a lo anterior, la siguiente pregunta resulta ineludible: ¿Qué anhelan ser cuando sean adultos? El riesgo de quedar atrapados en una ilusión virtual es alto, por lo que es necesario acercarlos desde ese plano a la situación real y objetiva.

Los jóvenes se enfrentan a las redes en forma natural y cotidiana sin pensar en la funcionalidad de las mismas; allí se insertan a través de la búsqueda, lo que los lleva a sustraerse cuando se activa el cliqueo para que los buscadores identifiquen ideas, como afirma Pariser (2017), quien llama a este proceso personalización, donde la mercadotecnia y los grandes del Internet están "predispuestos a secundar nuestras propias ideas. Tu pantalla de ordenador es cada vez más una especie de espejo unidireccional que refleja tus propios intereses, mientras los analistas de los algoritmos observan todo lo que clicas" (p. 13). Es fundamental que la escuela domine este tipo de información dirigido a cualquier cibernauta, 


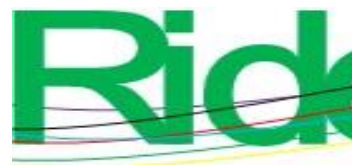

Revista Iberoamericana para la

Investigación y el Desarrollo Educativo

ISSN $2007-7467$

entre ellos los estudiantes, quienes se implican con el uso de sus datos e intereses por la información con las compañías virtuales, las cuales se dedican a cazar los motivos de búsqueda para después convertirse en ofertas seguras relacionadas con lo que el internauta ha encontrado previamente o tiene un mismo sentido. Es demandante asimismo analizar a la generación Nousmedi@ como concepto pedagógico, lo cual permite particionar en forma crítica lo que traen como referencia a las aulas para darle un contenido formativo con respecto a lo que la currícula escolar demanda, ya que es una realidad inevitable.

En este contexto, se propone considerar a la enseñanza desde tres enfoques pedagógicos que logren permear el mundo escolar para reorientar los trayectos escolares, y así los sujetos se perciban al ir construyendo su sentido presente-futuro. Los enfoques son los siguientes:

- $\quad$ Primeramente, al considerar indigno el olvidar la tarea de educar en diferentes ámbitos de la sociedad como la familia, la religión, la escuela, etc., al no hacer sinergia educativa para reflexionar, criticar y transformar con ellos todo aquello que aqueja al ser humano en su entorno social que se va olvidando. Para Freire (2012) "el mundo no es necesariamente esto o aquello, porque los seres humanos somos proyectos y al mismo tiempo podemos tener proyectos para el mundo" (p 47). Por otro lado, Han (2014, p. 14) menciona que "la indignación digital no puede cantarse" la reconoce como un estado afectivo que no desarrolla ninguna fuerza que genere algún poder de acción, aunque el contacto sea frecuente a través de las redes sociales, la relación se delimita, no se logra desarrollar ninguna fuerza poderosa que mueva sus conciencias. Y no conformar grupos sólidos con ideologías críticas genera gravitación, por lo que no engendra ningún futuro, pues se forma un enjambre digital sin un perfil propio. Advertir el lugar de los jóvenes en el movimiento de la vida y la experiencia formativa, donde la indignación no puede colocar a los sujetos en la responsabilidad y compromiso social, es la estrategia de trato o atención para reorientar o guiar el acto educativo.

- $\quad$ En segundo lugar, está la idea de ubicar a la vida cotidiana como el lugar donde cobra sentido la pedagogía, puesto que la condición humana pasa inexorablemente por ella. Aquí resuenan los conceptos de la ecopedagogía o pedagogía de la tierra, entendidos por Gadotti (2002) como el tener que reeducar la mirada de las personas en el reto desde sus primeros años escolares, lo cual implica un cambio completo en la forma de pensar la calidad de vida y el medio ambiente, sobre todo en el tipo de convivencia formativa con nosotros 


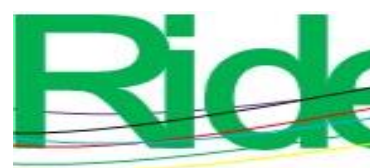

Revista Iberoamericana para la

Investigación y el Desarrollo Educativo

ISSN $2007-7467$

mismos, con los demás en el entorno natural. Así entra a escena la ecopedagogía, mediante una forma diferente a cuando se está enajenado en términos culturales, a partir de la vida cotidiana en un proceso que dé sentido a las cosas en apertura a nuevas sendas, con una vida auténtica de autocontrol no siendo extranjero de si, sino en la vivencia de la realidad, en el cuidado de sí mismo.

Es la educación cara a cara, y en su defecto por medios virtuales, donde la tarea se ha olvidado, el abandono de dar seguimiento y acompañamiento a los trayectos formativos de los estudiantes en la vinculación amorosa con la tierra, ese mundo donde respire, sienta, escuche, mire y exprese en el acontecer diario con los otros, formando parte de sí, del sujeto real y pensante al ver el deterioro. Reeducar y educar es un acto de trascender lo ineducable, ampliar la mirada pedagógica hacia la ciudadanía planetaria, y no solo en lo personal, como se indica en la currícula y la propuesta de reforma de la política educativa, documento que supone, en su meta 4.4: “de aquí a 2030, aumentar sustancialmente el número de jóvenes y adultos que tienen las competencias necesarias, en particular técnicas y profesionales, para acceder al empleo, el trabajo docente y el emprendimiento", difundida por la Organización de las Naciones Unidas para la Educación, la Ciencia y la Cultura [Unesco] (2015, p. 9). Sin embargo, para Gadotti (2002) se focaliza en lo humano cuando señala el "sentir, intuir, vibrar emocionalmente, imaginar, inventar, crear y recrear, relacionar e interconectarse, autoorganizarse, informarse, comunicarse, expresarse, localizar, procesar y utilizar la inmensa información de la aldea global; buscar causas y prever consecuencias, criticar, evaluar, sistematizar y tomar decisiones" (p. 162). Demanda reeducarnos como adultos para educar, reformar y ordenarse, exige volver a educarnos en los nuevos conocimientos para el nuevo mundo, sintiendo ese regreso en contacto con la naturaleza.

- Como tercera propuesta está la pedagogía de la ternura, del autor Cussiánovich (2005). Para hacer de la educación un bien social y un derecho irrenunciable, así como arrancarla del mercado en el que ha devenido en una mercancía, incidir en hacer conscientes a las nuevas generaciones que lo que hoy está en juego, es el proyecto de humanidad, el destino de la especie humana cuando se extiende la exclusión y se ahondan las desigualdades. Pensar a los niños en su propia vida, donde la ternura en la relación pedagógica se centre en su matriz epistemológica y existencial, refleja cómo se ha dejado al olvido dentro de las instituciones la tarea formadora con sentido social y política del hacer desde el presente el futuro de la humanidad, valores que se van confundiendo entre la practicidad tecnológica y 


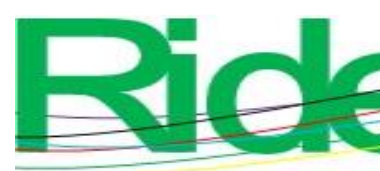

Revista Iberoamericana para la Investigación y el Desarrollo Educativo ISSN $2007-7467$

limitada realidad a un desconocimiento de la justicia, democracia, solidaridad, compromiso consigo mismo. El educar para la sociedad implica que se atiendan las necesidades tanto de índole social como las individuales que cada quien requiera.

Estas tres propuestas colocan al educador en situaciones, pedagogías y didácticas hacia el beneficio social y no de la situación laboral, sino como el desempeño proactivo profesional. Ser docente garante de formar parte de las generaciones ubicadas en el contexto virtual enriqueciendo al interior de las aulas, con la capacidad de indignación, del amor a la tierra (su planeta), la confianza en sí mismo y el otro en bien colectivo. Con estas demandas formativas se entremezclan pedagógicamente, en la comprensión del quehacer con el otro, no solo con las computadoras sino también, quien esté detrás de ella, como menciona Figueroa (2015), con "el smartphone y otros dispositivos móviles [que] están siendo utilizados recientemente dentro de las aulas de clase, tanto escolares como universitarias, [y a lo cual] se le denomina móvil learning, m-learning o aprendizaje móvil” (p.32). Sin duda el docente requiere aprender a considerar en su enseñanza lo que ya forma parte de la vida social.

Otra tarea docente en el trayecto escolar se fundamenta centralmente es la formación de una inteligencia asertiva (De la Plaza, 2012). Acompañados de un mentor que eduque con amor, en ese mundo de competitividad es necesario mejorar, primero, algunos esquemas mentales que en el mundo virtual se observan. Esta percepción se encuentra ante lo que se recibe desde la mercadotecnia en premios, reconocimiento social, facilitarse el pensar en un contexto de información ilimitada, recibir información que se ha cliqueado y no es el sujeto quien decide sino las grandes compañías que cuentan la mayoría de sus datos y preferencias, lo cual forma esquemas cognitivos poco asertivos, exitosos, rápidos, costosos, mayores grados académicos, mejor físico y estilo para vestir, entre otros que se agotan sin un impacto en la vida (Pariser, 2017). Aplicarlo con los estudiantes Nousmedi@ donde se defina una conducta asertiva es un continuo del trayecto escolar que debe estar presente en todas las acciones, máxime al decidir una profesión, la cual está relacionada con nuestro estilo personal y con lo que otorgue mayor capacidad de movilidad en diversas funciones. Se consideran cuatro rasgos según De la Plaza (2012) para una toma de decisión de tales dimensiones: 


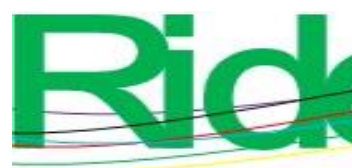

\section{Revista Iberoamericana para la Investigación y el Desarrollo Educativo ISSN $2007-7467$}

- Comprensión y definición muy clara del contenido a ser decidido, para no confundirse o agrandarlo en la mente.

- Análisis de sus posibles consecuencias, en el caso de optar por una u otra resolución.

- Síntesis de lo analizado, tanto en sus distintas facetas como en sus posibles consecuencias. Ejemplo: ¿Qué es lo peor que me podría pasar si me equivoco?

- $\quad$ Toma de decisión final y consecuente compromiso personal con ella (p. 154).

Es aquí que el papel del educador logra orientar por el futuro más certero al joven hacia la decisión de su carrera o campo laboral donde se sienta conectada y establecida la hibridación hombre-naturaleza-tecnología-cultura de una realidad factual, poniéndole en términos nuevamente de Benasayag (2015), hibridación para una realidad pedagógica en la que se construya la conexión con la realidad que las tecnologías han parcializado, para que el estudiante reconozca sus facultades y pueda identificar las demandas laborales posibles.

En relación con el ambiente laboral, Toche (23 de agosto de 2016) apunta lo siguiente:

Según los resultados de la encuesta Búsqueda de Empleo por Internet, de la Asociación Mexicana de Internet, 58 \% ha encontrado su empleo más reciente mediante las bolsas de trabajo en línea y $76 \%$ de los internautas confía en que su siguiente empleo lo encontrará por este medio (párr. 10).

También, en datos del Instituto Nacional de Estadística y Geografía [INEGI] (16 de mayo de 2018), se observa que el uso del Internet está asociado al nivel de estudios: entre más estudios mayor uso de la red. Estudiantes de educación superior usan internet en 94.5 $\%$, por lo que se establece como la interacción tecnológica durante el trayecto de cada grado a cursar hasta lograr una carrera profesional o un desempeño laboral.

Lo anterior permite comprender que las tecnologías de la información y la comunicación (TIC) aún no se han integrado como contenido educativo ya sea en las aulas o en plataformas virtuales a través del desarrollo humano para potenciar el mundo laboral.

El propósito central es avanzar hacia la construcción de neoaprendizaje para explicarnos y comprender en el proceso formativo que se experimenta en los medios digitales y cómo esto permite tomar decisiones sobre el trayecto formativo que se va eligiendo, de acuerdo con la información revisada. 


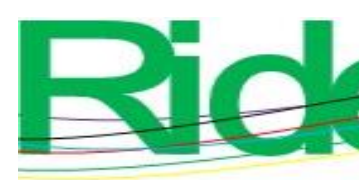

Revista Iberoamericana para la

Investigación y el Desarrollo Educativo

ISSN $2007-7467$

\section{Materiales y método}

Para abordar el seguimiento a los trayectos formativos de los estudiantes y recoger datos precisos y su experiencia con la tecnología durante sus estudios, el procedimiento metodológico fue de corte mixto integrando el enfoque cualitativo y cuantitativo a partir del fundamento de Buendía, Colás y Hernández (1998). Se utilizaron cuatro instrumentos: 1) un diario de campo, 2) un cuestionario, 3) los registros administrativos de los grupos universitarios y 4) el cárdex. El cuestionario constaba de 20 preguntas en total, la parte cualitativa con 9 preguntas abiertas y la cuantitativa con 11 preguntas cerradas con opción múltiple (sí, no y probablemente) y respuestas delimitadas correspondientes a indicadores precisos. El otro instrumento cuantitativo fueron las listas de datos: edad, carreras cursadas y grados académicos (semestres). Un último fue el cárdex escolar, donde se encontraron los promedios de aprovechamiento que han logrado en su educación superior. Se obtuvo información directa para el tratamiento de los resultados en los sujetos seleccionados que forman parte de la complejidad del objeto.

El muestreo intencional realizado fue por conveniencia o aleatorio, ya que todos los sujetos que componen la población cumplen con las mismas características, debido a que forman parte de una lista numerados. Fueron 97 niños y niñas identificados como Nousmedi@ dentro de la primera etapa de la investigación en el año de 2007, cuando cursaban el sexto grado de primaria. En esta segunda etapa, en el año 2018, utilizando la misma lista, son ahora jóvenes que se encontraban cursando entre tercero y octavo semestre de universidad o en instituciones formadoras de docentes, así como aquellos que pudieran haber desertado escolarmente. La aleatoriedad fue compleja debido a su ubicación, magnitud de la matrícula, la diversidad de las carreras y la flexibilidad de los programas universitarios en las diversas escuelas.

Las categorías que orientaron el procedimiento metodológico en la elaboración de ítems y resultados son:

- $\quad$ Estudiantes Nousmedi@.

- Trayecto formativo.

- $\quad$ Perfil profesional.

- Neoaprendizaje. 


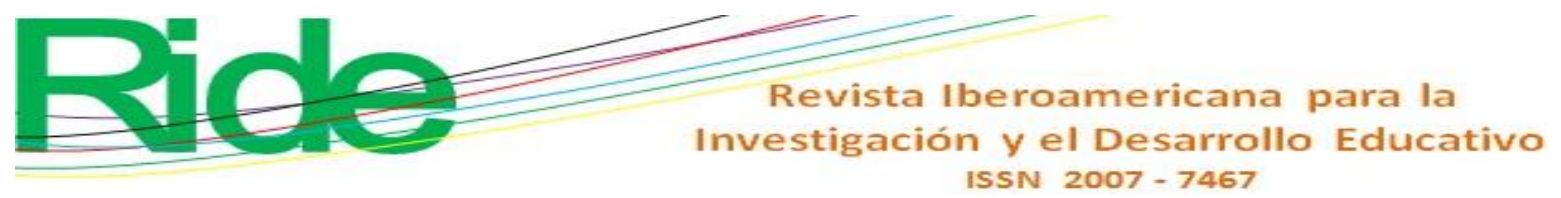

Trabajo de campo

El trabajo de campo se realizó de forma institucional. Posteriormente en lo individual con cada uno los estudiantes en las siguientes fases:

- $\quad$ Primera fase. Se procedió a nivel institucional enviando la lista de nombres a las diferentes universidades locales autónomas y privadas de Mexicali. Se realizó la gestión solicitando información sobre cada uno de ellos para dar paso a localizarlos físicamente.

- Segunda fase. Una vez localizados los jóvenes, se les contactó vía telefónica para averiguar con cada uno de ellos la disposición de ser encuestados. En el proceso se realizaron llamadas telefónicas y se enviaron correos electrónicos. Solo nueve aceptaron participar en ese periodo de tiempo, y quedó abierta la invitación abierta a quienes no tuvieron el tiempo suficiente.

- $\quad$ Tercera fase. La aplicación del cuestionario. De los nueve participantes, siete acudieron a la cita en un cubículo académico de la Universidad Pedagógica Nacional 021, en Mexicali, y a los otros tres se les visitó directamente previo acuerdo: una en su área de trabajo de diseño gráfico, otra como responsable de una farmacia de la localidad y otro en la universidad donde cursaba su carrera profesional, quien fue acompañado por el coordinador de la carrera. Se realizaron los protocolos de gestión institucionales previamente hasta abordarlos en persona.

- $\quad$ Cuarta fase. La sistematización de resultados de aprendizajes en sus trayectos escolares, entre los que fueron localizados en un grado académico, y se nos proporcionó por parte de la institución el nombre completo, edad, carrera profesional, grado de estudio, promedio, teléfono. Se organizaron los resultados del cuestionario y los promedios de calificación que han logrado.

- Quinta fase. La interpretación de resultados realizando la triangulación de la información, así como la redacción del documento académico. 


\section{Resultados y discusión}

Desde la primera etapa se realizó la gestión para solicitar a las autoridades correspondientes la información sobre los estudiantes detectados mediante listas de registro de inscripción. Este procedimiento dependía del tiempo de los responsables de área y la disposición para apoyar en el proyecto, que tuvo una duración de seis meses aproximadamente.

Después de la búsqueda de los 97 estudiantes, como ya se mencionó, se encontraron 66. Sobre este total de sujetos, a nueve se les pudo contactar por teléfono y después personalmente aplicar el cuestionario. Los estudiantes participaron no sin asombro por haber formado parte de una investigación cuando niños y también mostraron una gran disposición proporcionando la información solicitada.

Figura 1. Estudiantes Nousmedi@ localizados

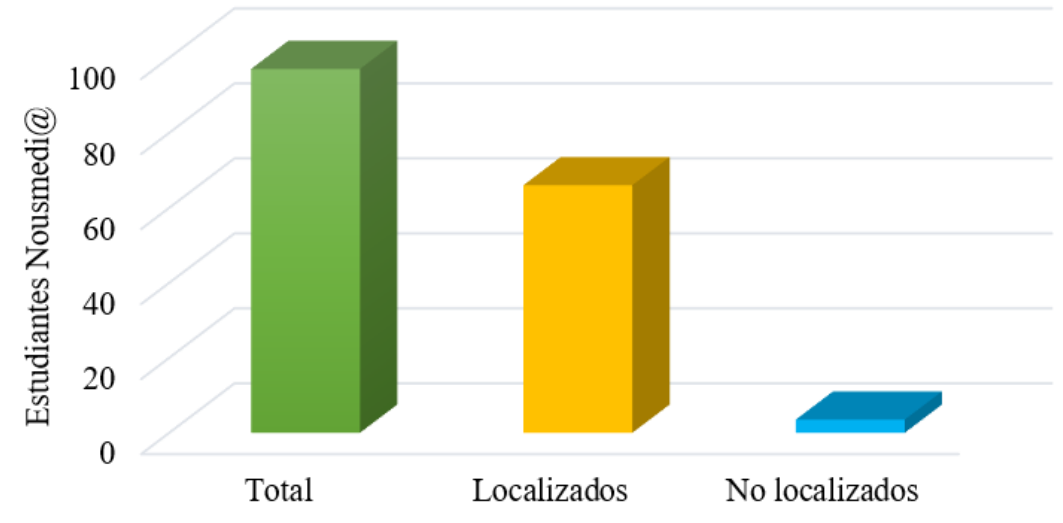

Fuente: Elaboración propia

Siguiendo los resultados de la figura 1, se encontraron en la muestra intencional de los 97 alumnos Nousmedi@ a 66 de estos, localizados a través de las instituciones públicas y privadas, lo cual arrojó los primeros datos de ubicación de la mayoría de ellos. Se puede observar asimismo que $68.4 \%$ fue ubicado como estudiantes en las universidades, en trabajo o egresados de la universidad, quienes siguieron su trayecto escolar. Por otro lado, $21.6 \%$ no fue localizado. Con esta información fue con la que se logró continuar la búsqueda hasta encontrarlos con alguno de los instrumentos. 
Figura 2. Forma de localización de los Nousmedi@

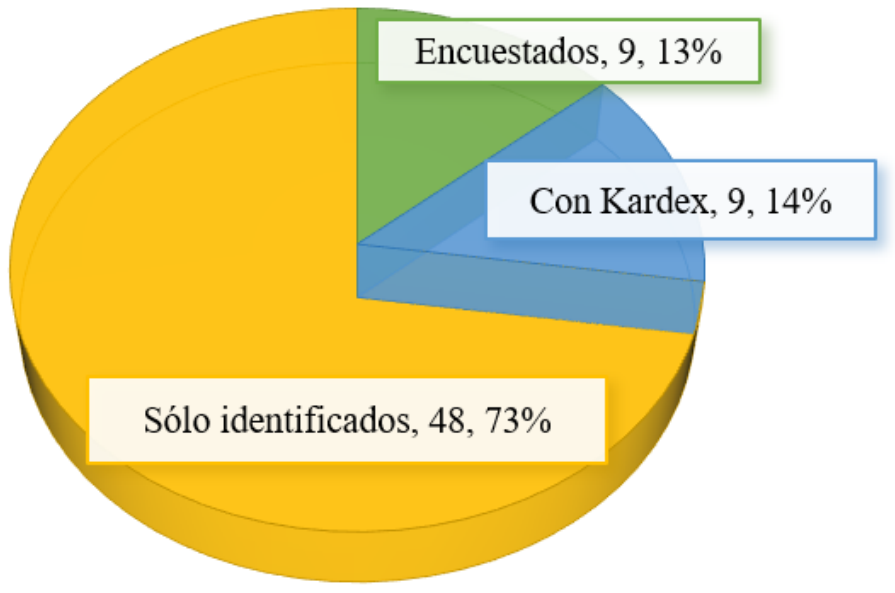

Fuente: Elaboración propia

En la figura 2 se observa que de los 66 estudiantes localizados solo nueve lograron ser encuestados, lo que representa un $9.13 \%$. Respecto al resto, se logró identificar en qué grado y carrera se encuentran. Se descubrió que $48.73 \%$ también siguió su trayecto escolar, por encontrarse en algún grado universitario, ya sea como egresado, activo o dado de baja. Por último, se contó con el cárdex de $9.14 \%$, dato que pudo ser contrastado con resultados que se muestran más adelante.

Figura 3. Nivel escolar logrado en el trayecto formativo

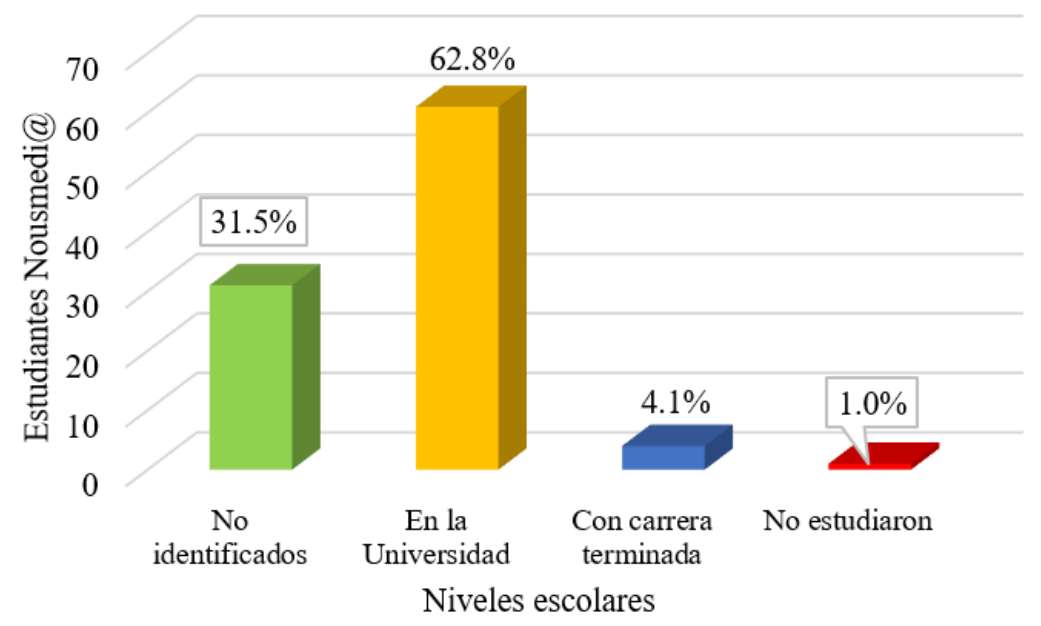

Fuente: Elaboración propia 


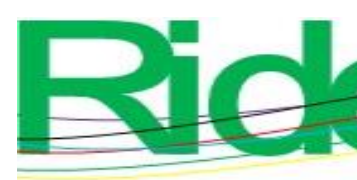

\section{Revista Iberoamericana para la Investigación y el Desarrollo Educativo ISSN $2007-7467$}

Ahora bien, tal y como se observa en la figura 3, de los 66 estudiantes localizados, 61 se encuentran estudiando en nivel universitario (62.8\%), solo cuatro han terminado su carrera profesional (4.1\%), y de estos cuatro, una se encuentra estudiando un posgrado en educación y dos estudiando una segunda carrera. El 1 \% representa solo a un estudiante Nousmedi@, quien fue localizado a través de llamada telefónica con el número que proporcionó cuando estudiaba sexto grado de primaria, y de quien se nos informó que no estudió, se casó y emigró a Estados Unidos, y ahora se desempeña en un trabajo técnico de soldador.

Figura 4. Perfiles profesionales del trayecto formativo del neoaprendizaje

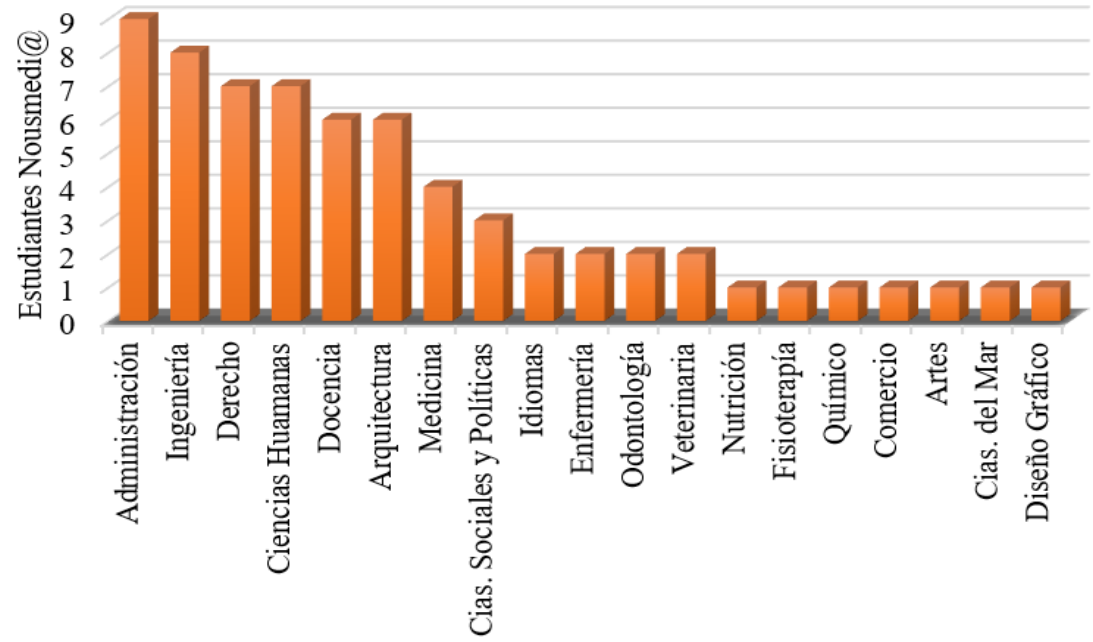

Fuente: Elaboración propia

Otro aspecto relevante para estos estudiantes Nousmedi@ relativo a su trayecto formativo es el de las diversas carreras que eligieron, la mayoría de ellas científicas y humanas. Se observa, entonces, que dentro de las carreras científicas se encuentran 37 estudiantes Nousmedi@ distribuidos de la siguiente forma: nueve en administración, ocho en ingeniería, seis en arquitectura, cuatro en medicina, dos en enfermería, dos en odontología, dos en veterinaria, uno en nutrición, uno en fisioterapia, uno en química y uno en ciencias del mar. Los otros 28 Nousmedi@ se ubican en carreras humanas: siete en derecho, siete en ciencias humanas, seis en docencia, tres en ciencias sociales y políticas, dos en idiomas, uno en artes, uno en comercio y uno en diseño gráfico (ver figura 4). Por lo que se observa que $57 \%$ presentó un mayor interés en su elección por carreras científicas y $43 \%$ decidieron cursar carreras humanísticas. 

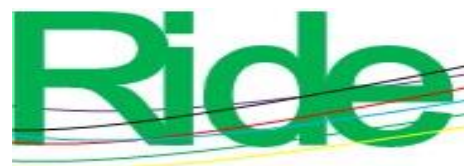

Revista Iberoamericana para la Investigación y el Desarrollo Educativo ISSN $2007-7467$

En estos resultados podemos observar la tendencia a elegir carreras con mayor relación al ámbito científico más que al humanístico, por lo que se puede decir que la influencia de la información recibida en las redes influye en la valoración de su desempeño a futuro. En el trayecto escolar, los estudiantes se encuentran ya con una realidad digitalizada y una comunicación en redes, interactuando tanto en el entorno virtual en el que aprendieron a construir desafíos o metas educativas como en el entorno escolar del deber ser social.

Figura 5. Tiempo dedicado a la cultura a través de la tecnología

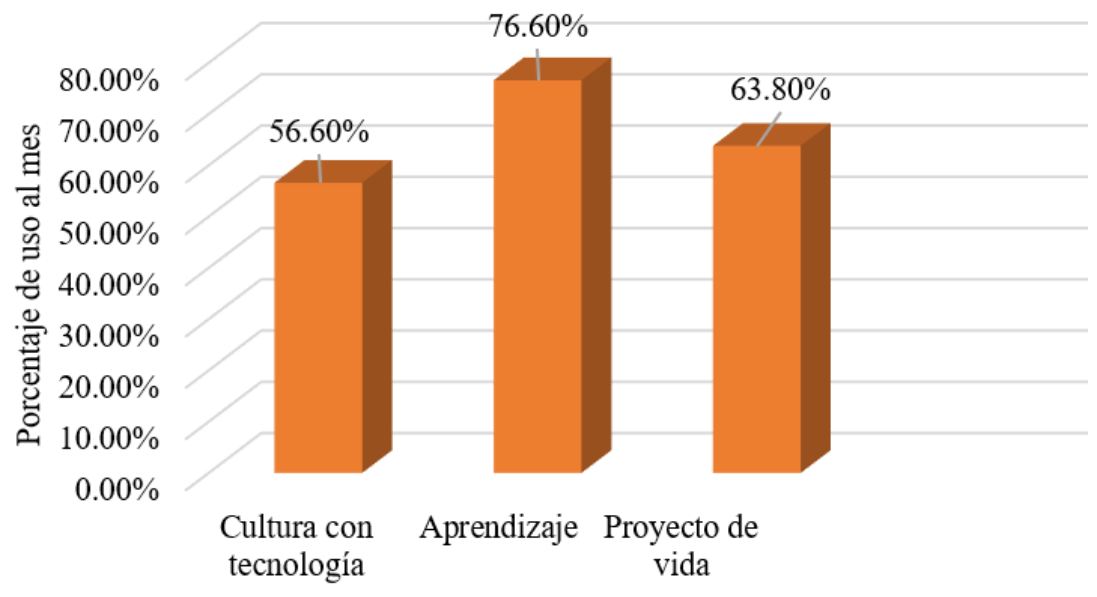

Fuente: Elaboración propia

En la figura 5, por su parte, se observa en un rango de 0 a 100 la forma en que los estudiantes confirman su manejo y dedicación de tiempo a la tecnología, con lo cual se genera el neoaprendizaje. La utilizan en $56.6 \%$ para lo cultural, $76.6 \%$ para el aprendizaje y un $63.80 \%$ para su proyecto de vida. Sin duda reconocen a la tecnología como un elemento que los acompaña de forma positiva a lo largo de su desarrollo escolar. 
Figura 6. Estrategias de aprendizaje que utilizas con tus compañeros para aprender

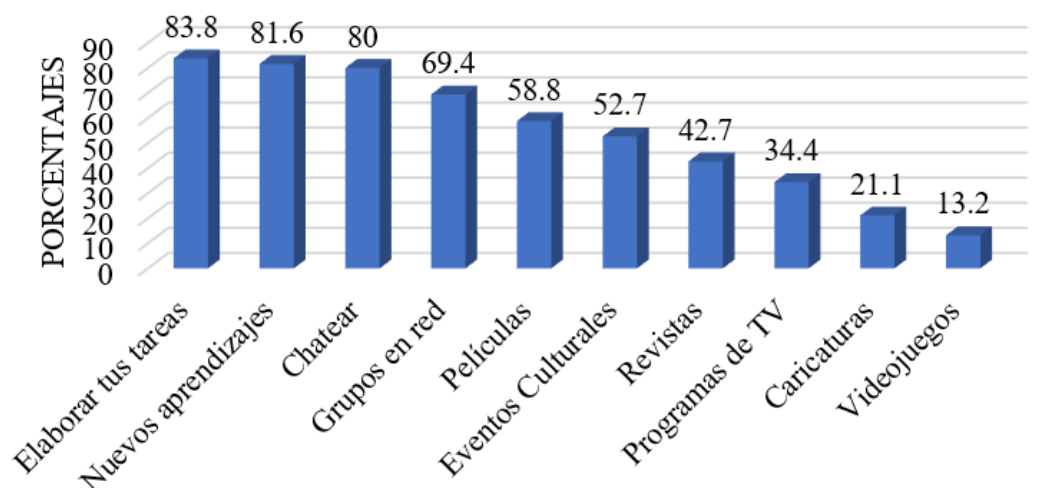

Fuente: Elaboración propia

El neoaprendizaje en su trayecto formativo se suscita en espacios fuera de la escuela y donde se comparte información sobre sus tareas y otros temas de interés. Se ubicó en la respuesta el nombre de varios dispositivos a través de los cuales los estudiantes interactúan previamente a las clases. Como se puede observar en la figura 6, hay respuestas organizadas con 10 dispositivos principales, de los cuales su uso de tecnología se explica con los siguientes resultados: para elaborar tareas encontramos $(83.8 \%)$, en la búsqueda y construcción de nuevos aprendizajes (81.6 \%), para chatear interactuando con sus compañeros (80 \%), la formación de grupos en Red (69.4\%), el uso de películas para estudio (58.8 \%), la participación en eventos culturales (52.7\%) y el uso de revistas (42.7\%). Como dispositivos de entretenimiento etiquetados a veces como distractores, se encuentran los programas de televisión (34.4\%), el mirar caricaturas (21.1\%) y los videojuegos (13.2\%), resultados que nos permiten ver cómo utilizan la tecnología en sus procesos de estudio y entretenimiento. La representación que dejan los datos es que los estudiantes pueden discernir y reflexionar el uso de la tecnología para sus actividades diarias, lo cual genera ciertos saberes que sirven de apoyo durante su trayecto formativo y en la toma de decisiones para consolidar su proyecto de vida.

Estos resultados se relacionan con una herramienta que actualmente ha dominado el manejo de la información y a la que Figueroa (2015) alude refiriendo a su vez a una investigación que "muestra que los estudiantes universitarios, usuarios de smartphones alcanzan un $97 \%$ \% (p. 31). Entonces, la variedad de usos confirma las habilidades que son cercanas a la elaboración de tareas y las que son distractores. Lo que determina que se cuenta con siete dispositivos de estudios con mayor de $40 \%$ de uso formativo, mientras que tres son de distracción y entretenimiento, con menos de $35 \%$ de uso. Esta categoría de 


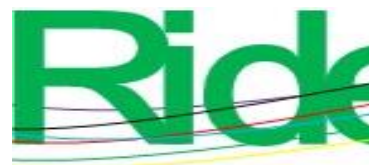

Revista Iberoamericana para la Investigación y el Desarrollo Educativo ISSN $2007-7467$

neoaprendizaje, de acuerdo con Banda (2017), se hace evidente como la construcción colectiva (dos o más dispositivos) de asimilar, aprehender y aprender compartiendo el contenido formativo previo y durante la clase.

La parte cualitativa del instrumento arroja que los estudiantes Nousmedi@ contactados y a quienes se les aplicó el cuestionario dejaron ver en sus respuestas que el uso de la tecnología fue en aumento a diferencia de cuando eran niños: ahora tienen mayor acceso a la misma y la han integrado a sus actividades cotidianas. Las respuestas indican lo siguiente:

- Utilizan la tecnología entre 7 y 20 horas al día.

- Utilizan aparatos como laptop, tabletas, celular y computadoras. Aunque respecto a la laptop, le dedican un mínimo en tiempo, que puede ser desde una hora. Y el más utilizado, eso sí, es el celular, conocido también como teléfono o smartphone, por unas 18 horas. La televisión no arrojó un parámetro fijo de uso.

- $\quad$ Opinan que pueden estudiar con la tecnología mediante la lectura, en forma auditiva, en interacción, comunicación, repaso, retroalimentación, facilitar el conocimiento, investigación, búsqueda de información solicitada y nueva, la obtención de datos, presentaciones, guías de palabras, punteo de ideas, exposiciones, mapas conceptuales, artículos científicos, modelos anatómicos y códigos de leyes, entre otros, lo que le da gran diversidad del uso tecnológico para su trayecto escolar.

- Les ayudan en forma textual y les facilita sus aprendizajes:

○ A través de grupos de Whatsapp y Messenger, que son marcas registradas de redes digitales que los jóvenes usan para comunicarse sobre sus tareas, discusión con compañeros por chat y búsqueda de conceptos, exposiciones, la comprensión de un tema nuevo, uso de videos sobre algún tema determinado.

○ Búsqueda e investigación de ideas, investigación en la Web de revistas científicas, ver el contraste de ideas, algún tema determinado como la importancia de la tecnología. Observar videos sobre embriología. Modelos anatómicos: saber cómo funciona el cuerpo y realizar cuadros sinópticos para fortalecer lo aprendido, punteo de ideas, precolección de información importante.

○ $\quad$ Es común el diseño y presentación de diapositivas (PowerPoint) para ciertos temas de la materia, sobre cómo llevar algo a cabo. 
- Leer la información para tener confianza en mí misma y poder desenvolverme de manera más segura, y así comprender adecuadamente y lograr dar su punto de vista de una manera más idónea.

○ Investigar antes de realizar alguna acción en el campo o a la realidad, experimentar la función con simuladores previos.

En la pregunta 12 del cuestionario indican que las formas de aprender con sus compañeros en las redes, previo a clases, facilita la interacción con la enseñanza que los profesores imparten en el aula y sus compañeros que no utilizan tecnología. Opinan que pueden comunicarse y ponerse de acuerdo para explorar un tema o disipar dudas, que es una herramienta fácil y cómoda, es una forma amena que puede mejorar en clase. Las redes son un medio para estar enterados sobre cuáles son los trabajos y compartir la información.

Otra de las preguntas centrales, la número 13, sobre el aprender los contenidos de la escuela a través de las redes virtuales que comparten con sus compañeros, arroja como respuesta que les facilita los resultados en el aula. Consideran que es más dinámico y fácil encontrar información y obtener conocimiento y ayudan entender mejor el tema. Se trabaja colaborativamente y en sincronía, hacer trabajos en grupo sin necesidad de estar presentes.

Reconocer que el aprender contenidos en la escuela a través de las redes virtuales les facilita expresar sus ideas, conocimientos y habilidades para definir su proyecto de vida. En la pregunta número 14 los participantes responden que todo se enlaza con la escuela, la vida cotidiana, para ser una persona completa, que todo gira alrededor de la tecnología, por lo que es una herramienta que ayuda a aprender de una manera sencilla y nos da una idea de cómo será, facilita lo que toma más tiempo. Debido a que le permite tener un amplio conocimiento y mantener al día de lo que está sucediendo, ellos dicen, que existen tipos variables de información y no es tan monótono como un libro, ayuda en tomar nuevos conocimientos para sus carreras, la información está más a su alcance y la pueden aprehender de manera más fácil, por lo tanto, la tecnología los acompaña a lo largo de su escolaridad.

En las respuestas que nos reflejan si el uso de las tecnologías es importante en las decisiones que toman los Nousmedi@ para su proyecto de vida, suscitadas por la pregunta 20, que es la pregunta de cierre, arroja que $56.1 \%$ considera que sí utilizan las tecnologías al tomar decisiones para construir su proyecto de vida. Estas decisiones se han presentado en diversas formas, como buscar estrategias e información, se actualizan constantemente y con la reconstrucción de las nuevas lógicas de realidad que configuran al complementarse en esta 
generación lejana a lo que la educación pretende para ellos. Investigan y accionan todas las posibilidades a su alcance digitalmente para llevarlo a cabo: cuál es la mejor opción, qué les conviene más y qué riesgos y frutos pueden lograr. Uno de ellos comenta que al contar con la tecnología se informó de la problemática de salud real en países subdesarrollados, lo cual influyó demasiado en la decisión de elegir la carrera de medicina (encuestado núm. 9). También reconocen que no toda la información que se encuentra en Internet es verdadera y que se tiene que saber dónde buscar. Hoy en día un simple mensaje o una llamada o en su defecto una videollamada puede influir mucho; un correo, por ejemplo, "nos avisa si fuimos o no" (encuestado núm. 9).

Los cárdex que se obtuvieron en forma impresa fueron permitidos para ser utilizados solo para documentar los promedios de aprendizaje. Se tomó únicamente el dato más alto y el más bajo para realizar la comparación de una distancia sobre 10 años de escolaridad. Todo esto se presenta en la tabla 1.

Tabla 1. Tabla comparativa de calificaciones 2007-2017

\begin{tabular}{|c|c|c|c|}
\hline $\begin{array}{c}\text { Nivel escolar y año } \\
\text { lectivo }\end{array}$ & Calificación baja & Calificación alta & $\begin{array}{c}\text { Diferencia a 10 } \\
\text { años }\end{array}$ \\
\hline 2007 & $\begin{array}{c}8.08 \\
\text { (equivalente en } \\
\text { centésimas a } \\
80.08)\end{array}$ & $\begin{array}{c}\text { (equivalente en } \\
\text { centésimas a 90.18) }\end{array}$ & 0.45 \\
\hline 2017 & 72.33 & 89.73 & 7.75 \\
\hline
\end{tabular}

Fuente: Elaboración propia

La tabla 1 muestra que cuando cursaban sexto grado de primaria en el año 2007 (97 alumnos), el promedio más alto fue de 9.18 y el más bajo 8.02 de calificación; ahora, en el 2017 (muestra de nueve alumnos), en nivel universitario, la calificación baja es de 72.33 y la más alta de 89.73. Para encontrar la diferencia a distancia de 10 años de escolaridad, se realizó la equivalencia en centésimas, y se observa una diferencia en su aprovechamiento escolar bajo de 7.75, lo cual resulta notoria tomando en cuenta que la diferencia en la calificación baja es sobre dos casos que se dieron de baja, los cuales representan la calificación menor. Sin embargo, en la calificación alta 0.45 la diferencia se mantiene y es 


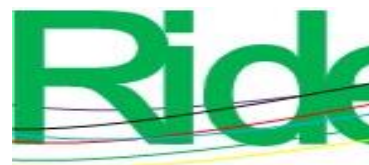

Revista Iberoamericana para la Investigación y el Desarrollo Educativo ISSN 2007 - 7467

poco notoria en los promedios parciales de los Nousmedi@. Por lo que, en la calificación general aprobatoria en esta muestra aleatoria, encontramos que continúan respondiendo positivamente a sus actividades en el logro aprendizaje.

A partir de todo ello se puede señalar cómo el mundo virtual se ha insertado en la enseñanza dentro de las aulas como medios de aprendizaje, pero aún no se logra la conceptualización que explique cómo aprenden las nuevas generaciones en su contexto digital, más aún si para ese contexto que ha creado un sendero que permea lo aprendido en días y horas que con anterioridad a sus clases obtienen para el tratamiento de un tema en específico. De ahí que no se ha dado una evolución en la enseñanza. Confirmamos que el aprendizaje que se pretende dentro de las aulas se interactúa en forma extraclase por los estudiantes en el universo digital, espacio donde constituyen el neoaprendizaje. Exclusivamente estos participantes conocen el contenido, el procedimiento y la finalidad de dicha interacción con el fin de preparar saberes previos e información requerida para tratar el aprendizaje con el docente y sus compañeros presencialmente.

\section{Conclusiones}

Partiendo de los datos ya obtenidos en la etapa exploratoria y al encontrar parte de los 97 estudiantes, la dificultad estuvo en que las autoridades de las universidades realizaran la gestión hacia las áreas administrativas. De hecho, en una de las universidades fue un proceso lento, ya que no se contaba con el personal para realizar dicha búsqueda. La fortaleza es que se obtuvo más de $50 \%$ de los datos para ubicarlos por escuelas. Al acudir a estas fue complicada la búsqueda por la flexibilidad de currícula y la ubicación por aulas y horarios específicos de cada estudiante. Así que se optó por las llamadas telefónicas.

Ya localizados los 65 estudiantes, solo nueve de ellos accedieron a darnos conocer cuál fue el alcance formativo o trayecto que han logrado en la escuela con las experiencias de ser un sujeto interactivo tecnológicamente. Se encontró que la mayoría de los estudiantes Nousmedi@ logró desarrollarse con los neoaprendizajes a través de los medios tecnológicos, los cuales ya forman parte de su trayecto escolar, lo que se complementa con las tareas que realizan en sus aulas, y los cuales el profesor no puede identificar hasta que genere el ambiente apto de convergencias, donde aporten todo aquello descubierto y lo relacionen con 


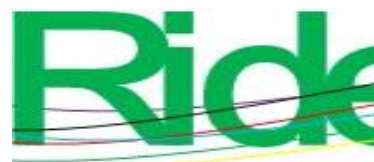

\section{Revista Iberoamericana para la \\ Investigación y el Desarrollo Educativo \\ ISSN $2007-7467$}

los contenidos de estudio. Esta interacción y vida en la interfaz les sirve para informarse de las oportunidades para continuar sus estudios o elegir el campo laboral acorde a su formación.

En suma, se encontró a quienes están estudiando todavía su carrera profesional, otros que ya la terminaron, y a una parte sin identificar actualmente. Sin embargo, $68.4 \%$ se encuentra en universidades. Y aunque no van en el grado académico que debieran, no han desistido en lograr una formación profesional.

En este encuentro se observó que los Nousmedi@ como a través del neoaprendizaje, logran reconocer su trayecto con ayuda de las redes sociales y los objetos de aprendizaje que se brindan en la interfaz, y pueden encontrar la información que los oriente a decidir un futuro profesional o laboral.

Por lo que se concluye que los rasgos característicos de los Nousmedi@ permiten reconceptualizar el aprendizaje en un sentido autoformativo, principalmente al momento en el que estos sujetos interactúan cliqueando con la tecnología, ya que activan y adquieren información, conocimientos, saberes, habilidades mentales, tecnológicas y socioculturales, gracias a lo cual forman un acervo mediático cibernético de la tecnocultura en una puesta en común con su semejante para un fin específico. A esto precisamente es a lo que llamamos neoaprendizaje, proceso que los profesores deben apropiarse para potencializar su quehacer formativo en las aulas.

La responsabilidad docente es comprender a dicha generación. Reconocemos que la generación son todos los sujetos que utilizan los medios digitales para informarse, construir sus saberes y compartir previamente a sus estudios académicos con lo encontrado, y así producir nuevas lógicas de análisis. Se trata, pues, de los Nousmedi@ con su forma característica que va del aprender al neoaprender.

Se concluye con el conocimiento de cuatro necesidades pedagógicas. Primero, reconocer que aquella educación que recupera el mundo de la interfaz desde la curiosidad de los estudiantes aporta a la formación de lo aquí llamado como cerebro aumentado. La cuestión es saber para qué se requiere dicho desarrollo, la exigencia formativa de hacer objetivo mediante las experiencias escolares y sociales que se relacionen con los contenidos curriculares. Segundo, que los docentes ameritan una formación pedagógica de evolución que refleje y guíe a sus estudiantes en el análisis crítico de la información y la realidad circundante: tener referencias en el mundo de la interfaz sobre cómo personalizan el uso del cliqueo para detectarse desde el mercado lo que deben consumir en producto o información, 


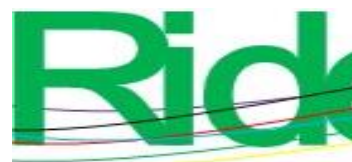

Revista Iberoamericana para la

Investigación y el Desarrollo Educativo

ISSN $2007-7467$

eso es un rescate de los sujetos que aprehenden en los medios digitales, quienes suelen alimentar los contenidos de estudio en las aulas. Tercero, los Nousmedi@ requieren ser regresados al planeta, es decir, del pensar al hacer, sentir la naturaleza, la vida, que comprendan que forman parte de una realidad que les afecta directamente en su desarrollo físico y en su salud mental. Por último, construir una pedagogía de la convergencia desde la docencia que consense con sus estudiantes el contenido de aprendizaje. Y abordar este trabajo con pedagogías identificadas con significados de la indignación, de la tierra y la del amor. Estos tres sentidos, que se articulan en la subjetividad tecnológica, y que se mantienen en olvido deben ser recuperados para la construcción didáctica. Este trabajo es un llamado a los docentes para reconceptualizar a sus estudiantes Nousmedi@ que no encuentran refugio formativo para la vida. La generación estudiada en el presente trabajo debió haber cumplido el total de grados escolares desde el año 2007, cuando estaban en sexto grado de primaria, y en 2018 estar terminando una carrera profesional. Ellos requirieron comprender que la escuela no había evolucionado como su contexto le demandaba, por ello es necesario estar construyendo propuestas de formación docente para atender estudiantes de forma convergente y en un sentido real.

\section{Agradecimientos}

A mi familia por estar siempre presente. En especial a mi esposo por creer en mis utopías y apoyar mis proyectos académicos. A mis hijos, quienes siguen el horizonte de la sabiduría rescatando lo mejor de la vida. A mis nietos y sobrinos de nuevas generaciones, quienes me inspiran para seguir en la búsqueda del eslabón educativo que parece perderse ante el avance de la tecnología.

Y al maestro Héctor Banda Prieto, maestrando de la University Texas A\&M, por su atinada colaboración en la revisión inicial de este trabajo. 


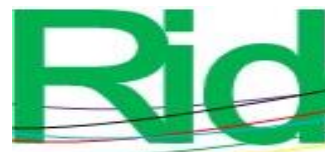

\section{Referencias}

Aronowitz, S., Martinsons, B. y Menser, M. (1998). Tecnociencia y cibercultura. La interrelación entre cultura, tecnología y ciencia. España: Paid Multimedia 7.

Banda, L. C. (2013). La inteligencia interactiva de los alumn@s nousmedi@.Un concepto para el andamiaje pedagógico del docente. México: Red de Investigadores de la Frontera.

Banda, L.C. (2017).L@s alumn@s nousmedi@: un concepto para comprender la enseñanza en las aulas del siglo XXI. México: Fondo Editorial del Magisterio.

Benasayag, M. (2015). El cerebro aumentado, el hombre disminuido. México: Paidós.

Buendía, E. L., Colás, B. P. y Hernández, P. F. (1998). La investigación por encuesta. En Métodos de investigación en psicopedagogía (pp. 119-155). México: McGraw-Hill.

Cussiánovich, A. (2005). Educando desde una pedagogía de la ternura. Perú. Recuperado de https://www.insumisos.com/lecturasinsumisas/Pedagogia\%20de\%201a\%20ternura .pdf.

De la Plaza, J. (2012). La inteligencia asertiva. Cómo, cuándo y dónde expresar lo que piensas. México: V\&R Editoras.

Figueroa, C. S. (2015). El uso del smartphone como herramienta para la búsqueda de información en los estudiantes de pregrado de educación de una universidad de Lima Metropolitana. Educación, 25(49). Recuperado de

Freire, P. (1997). Pedagogía de la autonomía. México: Siglo XXI.

Freire, P. (2012). La pedagogía de la indignación. España: Morata.

Gadotti, M. (2002). La Pedagogía de la Tierra. Argentina: Siglo XXI.

Han, Byung-Chul. (2014) El enjambre. Barcelona, España: Herder

Instituto Nacional de Estadística y Geografía [INEGI]. (16 de mayo de 2018). Comunicado de prensa núm. 208/18. México: Instituto Nacional de Estadística y Geografía. Recuperado de https://www.inegi.org.mx/contenidos/saladeprensa/aproposito/2018/internet2018_ Nal.pdf.

Organización de las Naciones Unidas para la Educación, la Ciencia y la Cultura [Unesco]. (2015). Declaración de Incheon. Educación 2030. Organización de las Naciones Unidas para la Educación, la Ciencia y la Cultura. Recuperado de

\section{(C)}




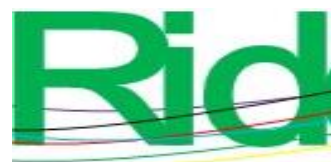

Revista Iberoamericana para la Investigación y el Desarrollo Educativo ISSN $2007-7467$

http://unesdoc.unesco.org/images/0023/002338/233813M.pdf.

Pariser, E. (2017). El filtro burbuja. Cómo la red decide lo que leemos y lo que pensamos. México: Taurus.

Quinta, J. M. (1993). Pedagogía Familiar. Recuperado de https://books.google.com.mx/books. Toche, N. (23 de agosto de 2016 ) Búsqueda de Trabajo por Internet la mejor. El Economista. Recuperado de https://www.eleconomista.com.mx/arteseideas/Busqueda-detrabajo-por-Internet-la-mejor-20160823-0106.html. 